Roman SADOWy (Opole)

\title{
ON RISK SENSITIVE CONTROL OF REGULAR STEP MARKOV PROCESSES
}

Abstract. Risk-sensitive control problem of regular step Markov processes is considered, firstly when the control parameters are changed at shift times and then in the general case.

1. Introduction. Let $E$ be a locally compact separable metric space, endowed with the Borel $\sigma$-algebra $\mathcal{E}$, and $U$ be a compact metric space with the Borel $\sigma$-algebra $\mathcal{U}$.

Given a stochastic kernel $P^{a}(x, B)$ which is a function defined for $(x, a) \in$ $E \times U$ and $B \in \mathcal{E}$ such that (i) for each $(x, a) \in E \times U, B \mapsto P^{a}(x, B)$ is a probability measure on $\mathcal{E}$, and (ii) for each $B \in \mathcal{E},(x, a) \mapsto P^{a}(x, B)$ is a measurable function on $E \times U$, and a function $\gamma: E \times U \rightarrow \mathbb{R}^{+}$, consider the following control problem. Suppose that there is a sequence of random moments, decision times, $\left(T_{n}\right)$ such that $T_{0}=0, T_{n}<T_{n+1}$ and $T_{n} \rightarrow \infty$ as $n \rightarrow \infty$. Initially, at time 0 we choose a control parameter $a_{0} \in U$, which is fixed in the interval $\left[T_{0}, T_{1}\right)$. At time $T_{n}$ we choose another control parameter $a_{n} \in U$, which is fixed in the interval $\left[T_{n}, T_{n+1}\right)$. This way we construct our control strategy $\bar{a}=\left(a_{t}\right)$, where $a_{t}=a_{n}$ for $T_{n} \leq t<T_{n+1}$.

The state process $\left(x_{t}\right)$ corresponding to the control strategy $\bar{a}$ starts from a point $x_{0} \in E$ and remains there for an exponentially distributed time $\sigma_{0}$ with parameter $\gamma\left(x_{0}, a_{t}\right)$. Then it is shifted to a new position $x_{1}$ according to the transition law $P^{a_{\sigma_{0}}}\left(x_{0}, \cdot\right)$. At $x_{1}$ it remains a time $\sigma_{1}$ which is exponentially distributed with parameter $\gamma\left(x_{1}, a_{t}\right), t \geq \sigma_{0}$, conditionally independent, given $x_{1}$, of $\sigma_{0}$. Then it is shifted to $x_{2}$ according to $P^{a_{\sigma_{1}}}\left(x_{1}, \cdot\right)$, and the procedure is repeated recursively.

Define $\tau_{0}:=0$, and $\tau_{n}:=\tau_{n-1}+\sigma_{n-1} \Theta_{\tau_{n-1}}, n=1,2, \ldots$, where $\Theta$ is a shift operator. Then for $t \geq 0$ we have $x_{t}=x_{n}$ for $\tau_{n} \leq t<\tau_{n+1}$.

2000 Mathematics Subject Classification: Primary 93E20; Secondary 60J75.

Key words and phrases: risk sensitive long run average cost criterion, regular step processes, Bellman equation. 
To describe evolution of the controlled process $X=\left(x_{t}\right)$ under the control stategy $\bar{a}=\left(a_{t}\right)$ we have to construct a probability space $\left(\Omega, \mathcal{F}, P^{\bar{a}}\right)$ and the corresponding filtration $\mathcal{F}_{t}$. For a detailed construction we refer the reader to [1].

Let $f: E \times U \rightarrow \mathbb{R}$ be a continuous bounded function. Our aim is to minimize the following long run average cost functional:

$$
J_{x}(\bar{a})=\frac{1}{\beta} \limsup _{t \rightarrow \infty} \frac{1}{t} \ln E_{x}^{\bar{a}}\left\{\exp \left(\beta \int_{0}^{t} f\left(x_{s}, a_{s}\right) d s\right)\right\},
$$

where $\beta>0$ is a constant and $E_{x}^{\bar{a}}$ denotes the expected value under the control stategy $\bar{a}$, given $x_{0}=x$, which means that the process $\left(x_{t}\right)$ starts from the point $x \in E$.

Denote by $C(E)$ (resp. $B(E)$ ) the space of continuous bounded (resp. bounded) real-valued functions on $E$ endowed with the uniform norm $\|\cdot\|$.

The following assumptions will be needed throughout the paper:

(A1) The mapping $E \times U \ni(x, a) \mapsto P^{a} f(x)=\int_{E} f(y) P^{a}(x, d y)$ is continuous for every $f \in C(E)$.

(A2) The function $(x, a) \mapsto \gamma(x, a)$ is continuous and there are constants $D>d>0$ such that $d \leq \gamma(x, a) \leq D$ for all $x \in E$ and $a \in U$.

$$
\beta<\frac{d}{2\|f\|} \text {. }
$$

The main results of the paper are formulated in the next two sections. In Section 2 we consider the case when the control parameters are changed at shift times only. We show the existence of a solution to the corresponding Bellman equation and the existence of an optimal strategy.

Section 3 establishes the relation between optimal control strategies with changes of control parameters at shift times and changes at arbitrary random moments.

2. Control at shift times. In this section we consider the control model with the restriction that the control parameters are changed at shift times only. Namely, in this case we choose the control parameters $a_{n}$ at times $T_{n}=\tau_{n}, n=0,1, \ldots$, and consequently let $a_{t}=a_{n}$ for $\tau_{n} \leq t<\tau_{n+1}$. We denote this strategy by $\widehat{a}$.

Consider the equation

$$
e^{\omega(x)}=\inf _{a \in U} E_{x}^{a}\left\{\exp \left(\int_{0}^{\tau} \beta\left[f\left(x_{s}, a_{s}\right)-\lambda\right] d s+\omega\left(x_{\tau}\right)\right)\right\},
$$

where $\tau$ is exponentially distributed with parameter $\gamma(x, a)$. The next proposition establishes the relationship between equation (2.1) and the optimal 
value of the functional (1.1). Moreover, this proposition provides information about an optimal strategy.

Proposition 2.1. If there exist a function $\omega \in C(E)$ and a constant $\lambda$ such that equation (2.1) is satisfied then under assumptions (A1), (A2),

$$
\lambda=\inf _{\widehat{a}} J_{x}(\widehat{a})=J_{x}\left(u\left(x_{t}\right)\right)
$$

where $u: E \rightarrow U$ is a Borel function for which the inf in (2.1) is attained.

Proof. Define

$$
e^{\omega_{1}(x, a)}:=E_{x}^{a}\left\{\exp \left(\int_{0}^{\tau} \beta\left[f\left(x_{s}, a_{s}\right)-\lambda\right] d s+\omega\left(x_{\tau}\right)\right)\right\} .
$$

Note that under assumptions $(A 1),(A 2)$ and $\omega \in C(E)$ the function $\omega_{1}(x, a)$ is continuous and bounded. Furthermore

$$
e^{\omega(x)}=\inf _{a \in U} e^{\omega_{1}(x, a)} \text {. }
$$

Moreover, we have

$$
e^{\omega_{1}(x, a)} \leq E_{x}^{a}\left\{\exp \left(\int_{0}^{\tau \wedge t} \beta\left[f\left(x_{s}, a_{s}\right)-\lambda\right] d s\right)\left[e^{\omega\left(x_{\tau}\right)} \chi_{\{\tau \leq t\}}+\chi_{\{\tau>t\}} e^{\omega_{1}\left(x_{t}, a_{t}\right)}\right]\right\} .
$$

By induction we obtain

$$
\begin{aligned}
e^{\omega_{1}(x, a)} \leq & E_{x}^{\left(a_{n}\right)}\left\{\exp \left(\int_{0}^{\tau_{n} \wedge t} \beta\left[f\left(x_{s}, a_{s}\right)-\lambda\right] d s\right)\right. \\
& \left.\times\left[\chi_{\left\{\tau_{n} \leq t\right\}} e^{\omega\left(x_{\tau_{n+1}}\right)}+\sum_{i=0}^{n-1} \chi_{\left\{\tau_{i} \leq t\right\}} \chi_{\left\{\tau_{i+1}>t\right\}} e^{\omega_{1}\left(x_{t}, a_{t}\right)}\right]\right\} .
\end{aligned}
$$

Note that under assumption $(A 2)$ we have $\tau_{n} \rightarrow \infty$ as $n \rightarrow \infty$. Thus letting $n \rightarrow \infty$ we obtain

$$
e^{\omega_{1}(x, a)} \leq E_{x}^{\widehat{a}}\left\{\exp \left(\int_{0}^{t} \beta\left[f\left(x_{s}, a_{s}\right)-\lambda\right] d s+\omega_{1}\left(x_{t}, a_{t}\right)\right)\right\}
$$

and

$$
\lambda \beta \leq \frac{1}{t} \ln E_{x}^{\bar{a}}\left\{\exp \left(\int_{0}^{t} \beta f\left(x_{s}, a_{s}\right) d s\right)\right\}+\frac{\left\|\omega_{1}\right\|-\omega_{1}(x, a)}{t} .
$$

Therefore letting $t \rightarrow \infty$ we get

$$
\lambda \leq \frac{1}{\beta} \limsup _{t \rightarrow \infty} \frac{1}{t} \ln E_{x}^{\bar{a}}\left\{\exp \left(\int_{0}^{t} \beta f\left(x_{s}, a_{s}\right) d s\right)\right\}
$$

with equality for the strategy for which the inf in (2.1) is attained. 
REMARK 2.1. It is easily seen that the constant $\lambda$ satisfying equation (2.1) is bounded. Namely,

$$
-\|f\| \leq \lambda \leq\|f\| .
$$

Therefore, to study the Bellman equation (2.1) it is sufficient to consider the case $|\lambda| \leq\|f\|$.

Consequently, the optimal control problem is reduced to the problem of the existence of a unique solution to (2.1). Notice that (2.1) can be rewritten in the following equivalent form:

$$
\omega(x)=\inf _{a \in U}\left[\ln \frac{\gamma(x, a)}{\gamma(x, a)-\beta[f(x, a)-\lambda]}+\ln \int_{E} e^{\omega(y)} P^{a}(x, d y)\right] .
$$

For $|\lambda| \leq\|f\|$ define

$$
g(x, a, \lambda):=\ln \frac{\gamma(x, a)}{\gamma(x, a)-\beta[f(x, a)-\lambda]} .
$$

Under assumptions $(A 2),(A 3), g$ is well defined, continuous and bounded. Moreover, $g$ is decreasing with respect to $\lambda$.

For $|\lambda| \leq\|f\|$ consider the auxiliary equation

$$
\widehat{\omega}_{\lambda}(x)+\widehat{\lambda}(\lambda)=\inf _{a \in U}\left[g(x, a, \lambda)+\ln \int_{E} e^{\widehat{\omega}_{\lambda}(y)} P^{a}(x, d y)\right],
$$

where $\widehat{\lambda}(\lambda)$ is a constant depending on the parameter $\lambda$.

Moreover, consider the following long run average cost criterion:

$$
I_{x}^{\lambda}(\widehat{a})=\limsup _{t \rightarrow \infty} \frac{1}{t} \ln E_{x}^{\widehat{a}}\left\{\exp \left(\int_{0}^{t} g\left(x_{s}, a_{s}, \lambda\right) d s\right)\right\} .
$$

The next proposition provides a description of the optimal value and an optimal strategy for the functional (2.4) and follows from Theorem 2.1 of [4].

Proposition 2.2. If there exist an $\widehat{\omega}_{\lambda} \in C(E)$ and a constant $\widehat{\lambda}(\lambda)$ such that equation (2.3) is satisfied then under assumptions $(A 1)-(A 3)$,

$$
\widehat{\lambda}(\lambda)=\inf _{\widehat{a}} I_{x}^{\lambda}(\widehat{a})=I_{x}^{\lambda}\left(u\left(x_{s}\right)\right)
$$

where $u: E \rightarrow U$ is a Borel function for which the inf in (2.3) is attained.

Now we shall study the problem of the existence of a solution to the Bellman equation (2.3).

We shall need the following additional assumptions:

(B1) There exists $\Delta<1$ such that for all $x, x^{\prime} \in E, a, a^{\prime} \in U$ and $B \in \mathcal{E}$,

$$
P^{a}(x, B)-P^{a^{\prime}}\left(x^{\prime}, B\right) \leq \Delta .
$$


(B2) There exists $\eta \in \mathcal{P}(E)$ and a Borel function $E \times E \times U \ni(x, y, a) \mapsto$ $p(x, y, a)$ such that for all $x \in E, a \in U$ and $B \in \mathcal{E}$,

$$
P^{a}(x, B)=\int_{B} p(x, y, a) \eta(d y)
$$

and

$$
\sup _{x, x^{\prime} \in E} \sup _{y \in E} \sup _{a \in U} \frac{p(x, y, a)}{p\left(x^{\prime}, y, a\right)}=K<\infty .
$$

For $h \in C(E)$ and $\lambda \in[-\|f\|,\|h\|]$ define the operator

$$
T^{\lambda} h(x)=\inf _{a \in U}\left[g(x, a, \lambda)+\ln \int_{E} e^{h(y)} P^{a}(x, d y)\right] .
$$

We shall use the so-called span norm contraction principle (see [2]). The next two propositions are Proposition 2 and Theorem 1 in [2]. Therefore we omit the proofs.

Proposition 2.3. Assume $(A 1)-(A 3)$ and $(B 1)$. Then for $|\lambda| \leq\|f\|$ the operator $T^{\lambda}$ is a local contraction in $C(E)$ endowed with the span norm

$$
\|h\|_{\mathrm{sp}}=\sup _{x \in E} h(x)-\inf _{y \in E} h(y),
$$

namely for each $M>0$, there exists a constant $\alpha(M)<1$ such that for each $h_{1}, h_{2} \in C(E)$ with $\left\|h_{1}\right\|_{\mathrm{sp}} \leq M$ and $\left\|h_{2}\right\|_{\mathrm{sp}} \leq M$ we have

$$
\left\|T^{\lambda} h_{1}-T^{\lambda} h_{2}\right\|_{\mathrm{sp}} \leq \alpha(M)\left\|h_{1}-h_{2}\right\|_{\mathrm{sp}} .
$$

Proposition 2.4. Assume $(A 1)-(A 3)$ and $(B 1),(B 2)$. Then for $|\lambda| \leq$ $\|f\|$ the operator $T^{\lambda}$ is a global contraction in the span norm in $C_{L}(E) \subset$ $C(E)$, where $C_{L}(E)$ is the set of continuous bounded functions with span norm bounded by $L=\|g\|+\ln K$.

REMARK 2.2. Notice that the contraction constant $\alpha(M)$ in Proposition 2.3 may be chosen independent of $\lambda$.

REMARK 2.3. Under the assumptions of Proposition 2.4, for each $\lambda \in$ $[-\|f\|,\|f\|]$ there is a unique (up to an additive constant) function $\widehat{\omega}_{\lambda} \in$ $C_{L}(E)$ and a constant $\hat{\lambda}(\lambda)$ such that

$$
\widehat{\omega}_{\lambda}-T^{\lambda} \widehat{\omega}_{\lambda}=\widehat{\lambda}(\lambda)
$$

Now we are ready to state the main result of this section.

THEOREM 2.1. Under assumptions $(A 1)-(A 3),(B 1)$ and $(B 2)$ there exist a unique constant $\lambda^{*}$ and a unique (up to an additive constant) function $\omega_{\lambda^{*}} \in C(E)$ for which the Bellman equation (2.1) is satisfied.

For the proof of the theorem we need an auxiliary lemma. 
Lemma 2.1. Suppose that assumptions $(A 1)-(A 3),(B 1)$ and $(B 2)$ are satisfied. Then the mapping $[-\|f\|,\|f\|] \ni \lambda \mapsto \widehat{\lambda}(\lambda)$ is continuous. Moreover, $\hat{\lambda}(\lambda)$ is a decreasing function.

Proof. The proof will be divided into 3 steps.

STEP 1. For $h \in C(E)$ and $|\lambda| \leq\|f\|$ consider

$$
T^{\lambda} h(x)=\inf _{a \in U}\left[g(x, a, \lambda)+\ln \int_{E} e^{h(y)} P^{a}(x, d y)\right] .
$$

Then for $\lambda, \lambda_{1} \in[-\|f\|,\|f\|]$ we have

$$
\begin{aligned}
\left|T^{\lambda} h(x)-T^{\lambda_{1}} h(x)\right| \leq & \mid \inf _{a \in U}\left[g(x, a, \lambda)+\ln \int_{E} e^{h(y)} P^{a}(x, d y)\right] \\
& -\inf _{a \in U}\left[g\left(x, a, \lambda_{1}\right)+\ln \int_{E} e^{h(y)} P^{a}(x, d y)\right] \mid \\
\leq & \sup _{a \in U}\left|g(x, a, \lambda)-g\left(x, a, \lambda_{1}\right)\right| \\
\leq & \ln \left[1+\beta\left|\lambda-\lambda_{1}\right| / \widetilde{b}\right]
\end{aligned}
$$

where $\widetilde{b}=d-2\|f\| \beta>0$. Therefore

$$
\left|T^{\lambda} h(x)-T^{\lambda_{1}} h(x)\right| \rightarrow 0 \quad \text { as } \lambda \rightarrow \lambda_{1}
$$

uniformly in $x \in E$.

STEP 2. Next we show that

$$
\left\|\widehat{\omega}_{\lambda}-\widehat{\omega}_{\lambda_{1}}\right\|_{\mathrm{sp}} \rightarrow 0 \quad \text { as } \lambda \rightarrow \lambda_{1} .
$$

In fact,

$$
\left\|\widehat{\omega}_{\lambda}-\widehat{\omega}_{\lambda_{1}}\right\|_{\mathrm{sp}} \leq\left\|\widehat{\omega}_{\lambda}-\left(T^{\lambda}\right)^{n} 0\right\|_{\mathrm{sp}}+\left\|\left(T^{\lambda}\right)^{n} 0-\left(T^{\lambda_{1}}\right)^{n} 0\right\|_{\mathrm{sp}}+\left\|\left(T^{\lambda_{1}}\right)^{n} 0-\widehat{\omega}_{\lambda_{1}}\right\|_{\mathrm{sp}},
$$

where 0 is the null function defined on $E$. By the span norm contraction principle, since the contraction constant can be chosen independent of $\lambda$ we have

$$
\left\|\widehat{\omega}_{\lambda}-\left(T^{\lambda}\right)^{n} 0\right\|_{\mathrm{sp}} \leq \varepsilon, \quad\left\|\left(T^{\lambda_{1}}\right)^{n} 0-\widehat{\omega}_{\lambda_{1}}\right\|_{\mathrm{sp}} \leq \varepsilon
$$

for $\varepsilon>0, \lambda$ close to $\lambda_{1}$ and sufficiently large $n$. Moreover, for a fixed $n$ we can choose $\lambda$ sufficiently close to $\lambda_{1}$ such that

$$
\left\|\left(T^{\lambda}\right)^{n} 0-\left(T^{\lambda_{1}}\right)^{n} 0\right\|_{\mathrm{sp}} \leq \varepsilon .
$$

STEP 3. Notice that by Step 2 there is a constant $p_{\lambda}$ such that

$$
\sup _{x \in E}\left|\widehat{\omega}_{\lambda}(x)-p_{\lambda}-\widehat{\omega}_{\lambda_{1}}(x)\right| \rightarrow 0 \quad \text { as } \lambda \rightarrow \lambda_{1} .
$$

Therefore for $x \in E$,

$$
\widehat{\omega}_{\lambda}(x)=p_{\lambda}+\widehat{\omega}_{\lambda_{1}}(x)+\varepsilon_{\lambda}(x),
$$


where $\varepsilon_{\lambda}(x) \rightarrow 0$ as $\lambda \rightarrow \lambda_{1}$ uniformly in $x \in E$. Then by Remark 2.3, for $x \in E$ we have

$$
\begin{aligned}
\widehat{\lambda}(\lambda)= & \left(T^{\lambda}-T^{\lambda_{1}}\right) \widehat{\omega}_{\lambda}(x)+T^{\lambda_{1}}\left(\widehat{\omega}_{\lambda_{1}}(x)+\varepsilon_{\lambda}(x)\right) \\
& -T^{\lambda_{1}} \widehat{\omega}_{\lambda_{1}}(x)-\varepsilon_{\lambda}(x)+\widehat{\lambda}\left(\lambda_{1}\right)
\end{aligned}
$$

and

$$
\begin{aligned}
\left|\widehat{\lambda}(\lambda)-\widehat{\lambda}\left(\lambda_{1}\right)\right| \leq & \left|\left(T^{\lambda}-T^{\lambda_{1}}\right) \widehat{\omega}_{\lambda}(x)\right|+\mid T^{\lambda_{1}}\left(\widehat{\omega}_{\lambda_{1}}(x)+\varepsilon_{\lambda}(x)\right) \\
& -T^{\lambda_{1}} \widehat{\omega}_{\lambda_{1}}(x)|+| \varepsilon_{\lambda}(x) \mid .
\end{aligned}
$$

Consequently

$$
\left|\widehat{\lambda}(\lambda)-\widehat{\lambda}\left(\lambda_{1}\right)\right| \rightarrow 0 \quad \text { as } \lambda \rightarrow \lambda_{1} .
$$

By Proposition 2.2 it is obvious that $\widehat{\lambda}$ is decreasing.

Proof of Theorem 2.1. By Remark 2.3, for $|\lambda| \leq\|f\|$ we have

$$
\widehat{\omega}_{\lambda}-T^{\lambda} \widehat{\omega}_{\lambda}=\widehat{\lambda}(\lambda)
$$

and $\widehat{\omega}_{\lambda} \in C(E)$. Since $\hat{\lambda}(-\|f\|)>0$ and $\widehat{\lambda}(\|f\|)<0$, there exists a unique constant $\lambda^{*}$ such that $\hat{\lambda}\left(\lambda^{*}\right)=0$ and, consequently, $\widehat{\omega}_{\lambda^{*}}-T^{\lambda^{*}} \widehat{\omega}_{\lambda^{*}}=0$. This completes the proof.

3. General control model. Consider the Bellman equation

$$
\inf _{a \in U}\left[A^{a} e^{\omega(x)}+e^{\omega(x)} \beta[f(x, a)-\lambda]\right]=0,
$$

where $A^{a}$ is a linear bounded operator defined on $B(E)$ and has the following explicit form:

$$
A^{a} h(x)=\gamma(x, a)\left[\int_{E}[h(y)-h(x)] P^{a}(x, d y)\right], \quad h \in B(E) .
$$

Notice that under assumptions $(A 1),(A 2), A^{a}: C(E) \rightarrow C(E)$.

The next proposition provides a criterion for an optimal value of functional (1.1) and an optimal strategy for the control problem.

Proposition 3.1. Assume $(A 1)$, (A2). If there exist a function $\omega \in$ $C(E)$ and a constant $\lambda$ such that equation (3.1) is satisfied, then

$$
\lambda=\inf _{\bar{a}} J_{x}(\bar{a})=J_{x}\left(u\left(x_{t}\right)\right)
$$

where $u: E \rightarrow U$ is a Borel function for which the inf in (3.1) is attained.

Proof. For $h \in C(E)$ define the following semigroup:

$$
\widehat{P}_{t} h(x)=E_{x}^{\left(a^{t}\right)}\left\{\exp \left(\int_{0}^{t} \beta\left[f\left(x_{s}, a_{s}\right)-\lambda\right] d s\right) h\left(x_{t}\right)\right\},
$$


where by $\left(a^{t}\right)$ we denote the restriction of the control strategy $\bar{a}=\left(a_{t}\right)$ up to time $t$. The corresponding generator, denoted by $\widehat{A}^{a}$, has the form

$$
\widehat{A}^{a}=A^{a} h(x)+h(x) \beta[f(x, a)-\lambda],
$$

where $A^{a}$ is as in (3.2). By (3.1) for $k, t \geq 0$ we have

$$
\begin{aligned}
0 \leq & \int_{T_{k} \wedge t}^{T_{k+1} \wedge t} \widehat{P}_{s} \widehat{A}^{a} e^{\omega(x)} d s \\
= & E_{x}^{\left(a^{t}\right)}\left\{\exp \left(\int_{0}^{T_{k+1} \wedge t} \beta\left[f\left(x_{s}, a_{s}\right)-\lambda\right] d s+\omega\left(x_{T_{k+1} \wedge t}\right)\right)\right\} \\
& -E_{x}^{\left(a^{t}\right)}\left\{\exp \left(\int_{0}^{T_{k} \wedge t} \beta\left[f\left(x_{s}, a_{s}\right)-\lambda\right] d s+\omega\left(x_{T_{k} \wedge t}\right)\right)\right\},
\end{aligned}
$$

and, consequently,

$$
\begin{aligned}
0 & \leq \sum_{k=0}^{n} \int_{T_{k} \wedge t}^{T_{k+1} \wedge t} \widehat{P}_{s} \widehat{A}^{a} e^{\omega(x)} d s \\
& =E_{x}^{\left(a^{t}\right)}\left\{\exp \left(\int_{0}^{T_{n+1} \wedge t} \beta\left[f\left(x_{s}, a_{s}\right)-\lambda\right] d s+\omega\left(x_{T_{n+1} \wedge t}\right)\right)\right\}-e^{\omega(x)} .
\end{aligned}
$$

Therefore, letting $n \rightarrow \infty$ we obtain

$$
e^{\omega(x)} \leq E_{x}^{\bar{a}}\left\{\exp \left(\int_{0}^{t} \beta\left[f\left(x_{s}, a_{s}\right)-\lambda\right] d s+\omega\left(x_{t}\right)\right)\right\},
$$

and, letting $t \rightarrow \infty$, we get

$$
\lambda \leq \frac{1}{\beta} \limsup _{t \rightarrow \infty} \frac{1}{t} \ln E_{x}^{\bar{a}}\left\{\exp \left(\int_{0}^{t} \beta f\left(x_{s}, a_{s}\right) d s\right)\right\},
$$

and equality holds for the control strategy for which the inf in (3.1) is attained.

Now, let $\widehat{a}=\left(a_{t}\right)$ be a strategy with changes of control parameters at shift times only. By (2.2) we have

$$
e^{\omega(x)} \leq \frac{\gamma(x, a)}{\gamma(x, a)-\beta[f(x, a)-\lambda]} \int_{E} e^{\omega(x, a)} P^{a}(x, d y) .
$$

Since $\gamma(x, a)-\beta[f(x, a)-\lambda]>0$, we get

$$
\gamma(x, a) \int_{E}\left[e^{\omega(y)}-e^{\omega(x)}\right] P^{a}(x, d y)+e^{\omega(x)} \beta[f(x, a)-\lambda] \geq 0
$$

with equality for the optimal strategy. 
Consequently the function $\omega$ and a constant $\lambda$ which are a solution to (2.1) also solve equation (3.1).

We summarize the above results in the following corollary.

COROLlary 3.1. The optimal value of the cost functional (1.1) over strategies with changes at shift times only is the same as the one for strategies with changes at arbitrary random moments.

Acknowledgments. The author wishes to express his thanks to Professor $€$. Stettner for suggesting the problem and for many stimulating conversations.

\section{References}

[1] R. M. Blumenthal and R. K. Getoor, Markov Processes and Potential Theory, Academic Press, New York, 1968.

[2] G. B. Di Masi and Ł. Stettner, Risk sensitive control of discrete time Markov processes with infinite horizon, SIAM J. Control Optim. 38 (2000), 61-78.

[3] E. B. Dynkin, Markov Processes, Springer, Berlin, 1965.

[4] D. Hernández-Hernández and S. J. Marcus, Risk sensitive control of Markov processes in countable state space, Systems Control Lett. 29 (1996), 147-155.

[5] O. Hernández-Lerma and J. B. Lasserre, Discrete-Time Markov Control Processes. Basic Optimality Criteria, Springer, New York, 1996.

Institute of Mathematics

Opole University

Oleska 48

45-052 Opole, Poland

E-mail: sadowy@math.uni.opole.pl 